VOL. 57 (1998) [295-303]

\title{
SPHERICAL SUBMANIFOLDS WITH SPECIAL QUADRIC REPRESENTATIONS
}

\author{
JITAN LU
}

\begin{abstract}
In this paper, we study submanifolds in the unit hypersphere satisfying $\Delta \widetilde{x}=$ $B \widetilde{x}+C$, where $\widetilde{x}$ is the quadric representation of the submanifold, and $B$ and $C$ are two constant matrices. We prove that the totally geodesic submanifolds are the only submanifolds in the unit hypersphere whose quadric representations satisfy $\Delta \tilde{x}=B \tilde{x}+C$.
\end{abstract}

\section{IntRoduction}

Let $x: M^{n} \rightarrow E^{m}$ be an isometric immersion of an $n$-dimensional Riemannian manifold into the $m$-dimensional Euclidean space, and $S M(m)$ be the space of the real symmetric matrices of order $m$. We define on $S M(m)$ the metric $g(P, Q)=$ $(\operatorname{tr}(P Q)) / 2$, for arbitrary $P, Q$ in $S M(m)$. Then this space becomes the standard $m(m+1) / 2$-dimensional Euclidean

transpose of $x$. Let $\tilde{x}=x x^{t}$. Then we obtain a smooth map $\widetilde{x}: M^{n} \rightarrow S M(m)$. Since the coordinates of $\widetilde{x}$ depend on the coordinates of $x$ in a quadric manner, we call $\widetilde{x}$ the quadric representation of $M^{n}[3]$. It is well known that for the hypersphere centred at the origin embedded in the Euclidean space in the standard way, the quadric representation is just the second standard immersion of the sphere. Then a question arises naturally: To what extent does the quadric representation of a submanifold in $E^{m}$ determine the submanifold? This question has been answered partly by many authors $[1,3,4,5,6]$. In $[3], 1$. Dimitric gave some results relative to the condition of $\tilde{x}$ being of finite type. In [5], the author proved that the only hypersurfaces with constant mean curvature which satisfy $\Delta \widetilde{x}=B \widetilde{x}+C$ are the hyperplane or the hypersphere centred at the origin. In this paper, we shall study submanifolds in the hypersphere of $E^{m}$ which satisfy the condition $\Delta \tilde{x}=B \tilde{x}+C$. We prove that the totally geodesic submanifolds are the only submanifolds in the unit hypersphere whose quadric representations satisfy $\triangle \tilde{x}=B \tilde{x}+C$.

Received 7th August, 1997

Copyright Clearance Centre, Inc. Serial-fee code: 0004-9729/98 SA2.00+0.00. 


\section{Preliminaries}

Let us fix the notation first. Let $x: M^{n} \rightarrow S^{m-1}(1) \subset E^{m}$ be an isometric immersion of an $n$-dimensional Riemannian manifold into the unit hypersphere $S^{m-1}(1)$ of the $m$-dimensional Euclidean space $E^{m}$. Then the position vector $x$ is a unit normal vector. We denote by $H$ the mean curvature vector of $M^{n}$ in $S^{m-1}(1)$. Let $e_{1}, \cdots, e_{n}$, $e_{n+1}, \cdots, e_{m-1}, x$ be local orthonormal vector fields along $M^{n}$, such that $e_{1}, \cdots, e_{n}$ are tangent to $M^{n}, e_{n+1}, \cdots, e_{m}$ are normal to $M^{n}$, and $e_{n+1}$ is parallel to $H$. Then $H=\alpha e_{n+1}$, where $\alpha$ is the mean curvature of $M^{n}$ in $S^{m-1}(1)$. Let $\langle$,$\rangle and \bar{\nabla}$ be the Euclidean metric and the connection of $E^{m}$, and denote by $\nabla, h, D, A_{r},\left|A_{r}\right|$ respectively, the connection of $M^{n}$, the second fundamental form of $M^{n}$ in $S^{m-1}(1)$, the normal connection of $M^{n}$ in $S^{m-1}(1)$, the Weingarten endomorphism relative to the normal direction $e_{r}$, and the length of $A_{r}, r=n+1, \cdots, m-1$.

In this setting, the indices $i, j, k$ always range from 1 to $n, r, s, t$ from $n+1$ to $m-1$ and $\beta, \gamma$ from $n+2$ to $m-1$.

We define a map $*$ from $E^{m} \times E^{m}$ into $S M(m)$ by $V * W=V W^{t}+W V^{t}$, for column vectors $V$ and $W$ in $E^{m}$. Then $V * W=W * V$. Let $\tilde{\nabla}$ denote the Euclidean connection of $S M(m)$, then we have

$$
\begin{aligned}
\tilde{\nabla}_{V}\left(W_{1} * W_{2}\right) & =\left(\bar{\nabla}_{V} W_{1}\right) * W_{2}+W_{1} *\left(\bar{\nabla}_{V} W_{2}\right), \\
g\left(V_{1} * V_{2}, W_{1} * W_{2}\right) & =\left\langle V_{1}, W_{1}\right\rangle\left\langle V_{2}, W_{2}\right\rangle+\left\langle V_{1}, W_{2}\right\rangle\left\langle V_{2}, W_{1}\right\rangle,
\end{aligned}
$$

and

$$
\Delta(V * W)=(\Delta V) * W+V *(\triangle W)-2 \sum_{i}\left(\bar{\nabla}_{e_{i}} V\right) *\left(\bar{\nabla}_{e_{i}} W\right)
$$

where $V, W, W_{1}, W_{2}, V_{1}$ and $V_{2}$ are all vectors in $E^{m}$, and $\Delta$ is the Laplacian operator of $M^{n}$ [3].

In this paper, we always denote by $X, Y$ and $Z$ tangent vectors of $M^{n}$, and by $V$ and $W$ column vectors in $E^{m}$.

\section{Minimal Submanifolds in the SPhere}

In this section, we consider the special case of a minimal submanifold.

Let $x: M^{n} \rightarrow S^{m-1}(1) \subset E^{m}$ be a minimal submanifold of $S^{m-1}(1)$. We denote the mean curvature vector of $M^{n}$ in $E^{m}$ by $\bar{H}$. Then

$$
\bar{H}=H-x=-x \text {. }
$$

But using (2.3) and (2.1), by a direct computation, we have

$$
\triangle \tilde{x}=-n \bar{H} * x-\sum_{i} e_{i} * e_{i}=n x * x-\sum_{i} e_{i} * e_{i} .
$$

Now we compute $\Delta^{2} \widetilde{x}$. 
Lemma 3.1. Let $x: M^{n} \rightarrow S^{m-1}(1) \subset E^{m}$ be a minimal submanifold of $S^{m-1}(1)$. Then

$$
\begin{aligned}
\triangle^{2} \widetilde{x}=2 n(n+1) x * x-2(n-1) & \sum_{i} e_{i} * e_{i} \\
& -2 \sum_{i, r}\left(A_{r} e_{i}\right) *\left(A_{r} e_{i}\right)+2 \sum_{r, s}\left(\operatorname{tr} A_{r} A_{s}\right) e_{r} * e_{s}
\end{aligned}
$$

Proof: Let $x$ be an arbitrary point in $M^{n}$; we may assume that $\nabla_{e_{i}} e_{j}=0$ at $x$. Then

$$
\begin{aligned}
\Delta e_{i} & =-\sum_{j} \bar{\nabla}_{e_{j}}\left(\bar{\nabla}_{e_{j}} e_{i}\right) \\
& =-\sum_{j} \bar{\nabla}_{e_{j}}\left(h\left(e_{i}, e_{j}\right)-\left\langle e_{i}, e_{j}\right\rangle x\right) \\
& =\sum_{j} A_{h\left(e_{i}, e_{j}\right)} e_{j}-\sum_{j} D_{e_{j}} h\left(e_{i}, e_{j}\right)-e_{i} .
\end{aligned}
$$

Since

$$
\sum_{j} A_{h\left(e_{i}, e_{j}\right)} e_{j}=\sum_{r} A_{r}^{2} e_{i}
$$

and

$$
\sum_{j} D_{e_{j}} h\left(e_{i}, e_{j}\right)=\sum_{j} D_{e_{i}} h\left(e_{j}, e_{j}\right)=0
$$

we have

$$
\sum_{i}\left(\Delta e_{i}\right) * e_{i}=\sum_{i, r}\left(A_{r} e_{i}\right) *\left(A_{r} e_{i}\right)-\sum_{i} e_{i} * e_{i}
$$

On the other hand,

$$
\sum_{i, j}\left(\bar{\nabla}_{e_{j}} e_{i}\right) *\left(\bar{\nabla}_{e_{j}} e_{i}\right)=\sum_{i, j} h\left(e_{i}, e_{j}\right) * h\left(e_{i}, e_{j}\right)+n x * x
$$

Combining the last two relations with (2.3), we obtain

$$
\begin{aligned}
\sum_{i} \triangle\left(e_{i} * e_{i}\right)=2 \sum_{i, r}\left(A_{r} e_{i}\right) *\left(A_{r} e_{i}\right)-2 \sum_{i} e_{i} * e_{i} \\
-2 \sum_{i, j} h\left(e_{i}, e_{j}\right) * h\left(e_{i}, e_{j}\right)-2 n x * x .
\end{aligned}
$$

Then from (3.1) and the last relation, we obtain (3.2). 
Theorem 3.2. Let $x: M^{n} \rightarrow S^{m-1}(1) \subset E^{m}$ be a minimal submanifold of $S^{m-1}(1)$. If its quadric representation satisfies $\Delta \widetilde{x}=B \widetilde{x}+C, M^{n}$ must be a totally geodesic submanifold of the unit hypersphere.

Proof: Let $x: M^{n} \rightarrow S^{m-1}(1) \subset E^{m}$ be a minimal submanifold of $S^{m-1}(1)$. If the quadric representation of $M^{n}$ satisfies the condition $\triangle \widetilde{x}=B \widetilde{x}+C$, from (3.1) we have

$$
\sum_{i} e_{i} * e_{i}-n x * x+B \widetilde{x}+C=0
$$

Differentiating the above relation along $X$, an arbitrary tangent vector of $M^{n}$, we have

$$
(B X) x^{t}+(B x) X^{t}-2(n+1) X * x+2 \sum_{r}\left(A_{r} X\right) * e_{r}=0 .
$$

Finding the $e_{r} * x$ and $e_{r} * Y$ components of (3.2) respectively, we have

$$
\left\langle B X, e_{r}\right\rangle=0,
$$

and

$$
\left\langle B x, e_{r}\right\rangle=0 .
$$

From (3.1) we know that

$$
B(\triangle \widetilde{x})=2 n(B x) x^{t}-2 \sum_{i}\left(B e_{i}\right)\left(e_{i}\right)^{t} .
$$

Now using (3.4) and (3.5), we find the $e_{t} * e_{t}$ components of (3.6) and (3.2) respectively. These are

$$
\left\langle B(\triangle \tilde{x}), e_{t} * e_{t}\right\rangle=0
$$

and

$$
\left\langle\triangle^{2} \widetilde{x}, e_{t} * e_{t}\right\rangle=4\left|A_{t}\right|^{2} .
$$

Since $\Delta \widetilde{x}=B \tilde{x}+C$, we know that $\Delta^{2} \widetilde{x}=\tilde{x}=B(\Delta \widetilde{x})$. Combining this relation with the above equations, we obtain $\left|A_{t}\right|^{2}=0$, for $t=n+1, \ldots, m-1$. This means that $M^{n}$ is a totally geodesic submanifold of the unit hypersphere.

\section{Submanifolds in the UNIT HyPERSPHERE}

In this section, we shall prove the following main result:

THEOREM 4.1. Let $x: M^{n} \rightarrow S^{m-1}(1) \subset E^{m}$ be a submanifold of $S^{m-1}(1)$. If its quadric representation satisfies $\Delta \widetilde{x}=B \tilde{x}+C, M^{n}$ must be a totally geodesic submanifold of the unit hypersphere.

The proof is lengthy; we divide it into a few lemmas. 
Lemma 4.2. Let $x: M^{n} \rightarrow S^{m-1}(1) \subset E^{m}$ be a submanifold of $S^{m-1}(1)$. If its quadric representation satisfies $\triangle \tilde{x}=B \tilde{x}+C$, there exists a function $\lambda(x)$ such that $\langle B X, Y\rangle=\lambda(x)\langle X, Y\rangle$ for any $X, Y \in T_{x}\left(M^{n}\right)$, the tangent space at an arbitrary point $x$ of $M^{n}$.

Proof: First, using (2.1) and (2.3), we compute the Laplace of $\tilde{x}$, that is

$$
\triangle \tilde{x}=n x * x-n \alpha e_{n+1} * x-\sum_{i} e_{i} * e_{i}
$$

Since $\Delta \widetilde{x}=B \widetilde{x}+C$, we have

$$
n \alpha e_{n+1} * x-n x * x+\sum_{i} e_{i} * e_{i}+B \widetilde{x}+C=0 .
$$

Differentiating this relation along any tangent vector $X$ of $M^{n}$, we obtain

$$
\begin{array}{rl}
0=(B X) x^{t}+2(n+1) X & * x+(B x) X^{t}+2 \sum_{r}\left(A_{r} X\right) * e_{r}+n X(\alpha) e_{n+1} * x \\
& +n \alpha e_{n+1} * X-n \alpha\left(A_{n+1} X\right) * x+n \alpha\left(D_{X} e_{n+1}\right) * x
\end{array}
$$

Finding the $e_{n+1} * Y$ component of $(4.2)$, we have

$$
\left\langle B x, e_{n+1}\right\rangle\langle X, Y\rangle+2 n \alpha\langle X, Y\rangle+4\left\langle A_{n+1} X, Y\right\rangle=0 .
$$

Then

$$
\left\langle A_{n+1} X, Y\right\rangle=\alpha\langle X, Y\rangle
$$

and

$$
\left\langle B x, e_{n+1}\right\rangle+2(n+2) \alpha=0 .
$$

But finding the $Y * x$ component of (4.2), we obtain

$$
\langle B X, Y\rangle+\langle B x, x\rangle\langle X, Y\rangle-2 n \alpha\left\langle A_{n+1} X, Y\right\rangle+4(n+1)\langle X, Y\rangle=0 .
$$

Combining (4.4) and (4.6), we have

$$
\langle B X, Y\rangle=\lambda(x)\langle X, Y\rangle,
$$

where $\lambda(x)=2 n \alpha^{2}-\langle B x, x\rangle-4(n+1)$. 
Lemma 4.3. Let $x: M^{n} \rightarrow S^{m-1}(1) \subset E^{m}$ be a full submanifold of $S^{m-1}(1)$. Then there does not exist a vector $V_{0} \in E^{m}$, which is normal to the tangent space $T_{x}\left(M^{n}\right)$ of $M^{n}$, for any $x \in M^{n}$.

We note that $M^{n}$ is a full submanifold of $S^{m-1}(1)$ means that (any piece of ) $M^{n}$ can not be contained in a lower dimensional linear subspace of $E^{m}$.

Proof: Suppose there exists a vector $V_{0} \in E^{m}$, which is normal to the tangent space $T_{x}\left(M^{n}\right)$ of $M^{n}$, for any $x \in M^{n}$. Then for any $X \in T_{x}\left(M^{n}\right)$, and any $x \in M^{n}$, we have $X\left\langle V_{0}, x\right\rangle=0$. This means $\left\langle V_{0}, x\right\rangle$ is a constant. Let $x_{0}$ be arbitrary point in $M^{n}$. Then $\left\langle V_{0}, x-x_{0}\right\rangle=0$. This means that $M^{n}$ is contained in the linear subspace

$$
\Lambda=\left\{W \in E^{m} ;\left\langle W, X_{0}\right\rangle=0\right\} .
$$

This is a contradiction to the fact that $M^{n}$ is full in $S^{m-1}(1)$.

Lemma 4.4. Let $x: M^{n} \rightarrow S^{m-1}(1) \subset E^{m}$ be a full submanifold of $S^{m-1}(1)$. Then there exist finite points $x_{1}, \ldots, x_{k}$, such that $T_{x_{i}}\left(M^{n}\right) \cap T_{x_{i+1}}\left(M^{n}\right) \neq\{0\}$, for $i=1, \ldots, k-1$ and $E^{m}=\operatorname{span}\left(\bigcup_{i=1}^{k} T_{x_{i}}\left(M^{n}\right)\right)$, where $\operatorname{span}\left(\bigcup_{i=1}^{k} T_{x_{i}}\left(M^{n}\right)\right)$ is the linear space obtained by spanning linearly from all the vectors in $\bigcup_{i=1}^{k} T_{x_{i}}\left(M^{n}\right)$.

Proof: Let $x_{1}$ be any point in $M^{n}$. It is impossible that $T_{x}\left(M^{n}\right)=T_{x_{1}}\left(M^{n}\right)$ always holds for any other point $x \in M^{2}$. For if it did, $M^{n}$ must be a $n$-dimensional Euclidean space. On the other hand, if $T_{x}\left(M^{2}\right) \cap T_{x_{1}}\left(M^{2}\right)=\{0\}$ always holds for any other point $x\left(\neq x_{1}\right) \in M^{2}$, we can choose any vector $X_{0} \in T_{x_{1}}\left(M^{2}\right)$. Then $X_{0}$ is normal to $T_{x}\left(M^{2}\right)$, for any other point $x$. It is obvious that $M^{n}-\left\{x_{1}\right\}$ is also a full submanifold in $S^{m-1}(1)$. From Lemma 4.3 we obtain a contradiction. Thus there exists $x_{2} \in M^{2}$, such that $T_{x_{1}}\left(M^{2}\right) \cap T_{x_{2}}\left(M^{2}\right) \neq\{0\}$ and $T_{x_{1}}\left(M^{n}\right) \neq T_{x_{2}}\left(M^{n}\right)$. It is obvious that the dimension of $\operatorname{span}\left(T_{x_{1}}\left(M^{n}\right)\right)$ is less than the dimension of $\operatorname{span}\left(\bigcup_{i=1}^{2} T_{x_{i}}\left(M^{n}\right)\right)$. Now the first $t$ points $x_{1}, \ldots, x_{t}$, which satisfy that $T_{x_{i}}\left(M^{n}\right) \cap T_{x_{i+1}}\left(M^{n}\right) \neq\{0\}$, for $i=1, \ldots, t-1$, and the dimension of $\operatorname{span}\left(\bigcup_{j=1}^{i} T_{x_{j}}\left(M^{n}\right)\right)$ is less than the dimension of $\operatorname{span}\left(\bigcup_{j=1}^{i+1} T_{x_{j}}\left(M^{n}\right)\right)$, have been defined. If the dimension of $\operatorname{span}\left(\bigcup_{j=1}^{t} T_{x_{j}}\left(M^{n}\right)\right)=m$, the proof is complete. If not, we can always choose a point $y \in M^{n}$, such that $T_{y}\left(M^{n}\right)$ is not contained in $\operatorname{span}\left(\bigcup_{j=1}^{t} T_{x_{j}}\left(M^{n}\right)\right)$. Since $T_{x}\left(M^{n}\right)$ changes continuously when $x$ changes, we can choose a point $x_{t+1} \in M^{n}$, such that $T_{x_{t}}\left(M^{n}\right) \cap T_{x_{t+1}}\left(M^{n}\right) \neq\{0\}$, and the dimension of $\operatorname{span}\left(\bigcup_{j=1}^{t} T_{x_{j}}\left(M^{n}\right)\right)$ is less than the dimension of $\operatorname{span}\left(\bigcup_{j=1}^{t+1} T_{x_{j}}\left(M^{n}\right)\right)$. By induction, 
we can choose finite points $x_{1}, \ldots, x_{k}$, such that $\left(\bigcup_{j=1}^{i} T_{x_{j}}\left(M^{n}\right)\right) \cap T_{x_{i+1}}\left(M^{n}\right) \neq\{0\}$, for $i=1, \ldots, k$ and $E^{m}=\operatorname{span}\left(\bigcup_{i=1}^{k} T_{x_{i}}\left(M^{n}\right)\right)$.

Lemma 4.5. Let $x: M^{n} \rightarrow S^{m-1}(1) \subset E^{m}$ be a full submanifold of $S^{m-1}(1)$. If there exists.a function $\lambda(x)$, such that $\langle B X, Y\rangle=\lambda(x)\langle X, Y\rangle$ for any $X, Y \in T_{x}\left(M^{n}\right)$ and any $x \in M^{n}$, the function $\lambda(x)$ must be a constant.

Proof: From Lemma 4.3, we can choose finite points $x_{1}, \ldots, x_{k}$, such that $\bigcup_{j=1}^{i} T_{x_{j}}\left(M^{n}\right) \cap T_{x_{i+1}}\left(M^{n}\right) \neq\{0\}$, for $i=1, \ldots, k-1$ and $E^{m}=\operatorname{span}\left(\bigcup_{i=1}^{k} T_{x_{i}}\left(M^{n}\right)\right)$. Since $T_{x_{1}}\left(M^{n}\right) \cap T_{x_{2}}\left(M^{n}\right) \neq\{0\}$, we can choose an non-zero vector $X \in T_{x_{1}}\left(M^{n}\right) \cap$ $T_{x_{2}}\left(M^{n}\right)$. It is obvious that $\lambda\left(x_{1}\right)\langle X, X\rangle=\lambda\left(x_{2}\right)\langle X, X\rangle$. Then $\lambda\left(x_{1}\right)=\lambda\left(x_{2}\right)$. Similarly, we obtain $\lambda\left(x_{1}\right)=\ldots=\lambda\left(x_{k}\right)$. Let $x$ be an arbitrary point of $M^{n}$. Since $E^{m}=\operatorname{span}\left(\bigcup_{i=1}^{k} T_{x_{i}}\left(M^{n}\right)\right)$, we know that $T_{x}\left(M^{n}\right) \cap\left(\bigcup_{i=1}^{k} T_{x_{i}}\left(M^{n}\right)\right) \neq\{0\}$. Then we can obtain $\lambda(x)=\lambda\left(x_{1}\right)=\ldots=\lambda\left(x_{k}\right)$. That is to say, $\lambda(x)$ is a constant.

LEMMA 4.6. Let $x: M^{n} \rightarrow S^{m-1}(1) \subset E^{m}$ be a full submanifold of $S^{m-1}(1)$. If its quadric representation satisfies $\Delta \widetilde{x}=B \widetilde{x}+C, M^{n}$ must be minimal.

Proof: From Lemma $4.2,4.3$ and 4.4 we know that $\lambda(x)=2 n \alpha^{2}-\langle B x, x\rangle-$ $4(n+1)$ is a constant.

Moreover, finding the $e_{j} * e_{k}$ component of (4.2), we have

$$
\left\langle B x, e_{j}\right\rangle\left\langle X, e_{k}\right\rangle+\left\langle B x, e_{k}\right\rangle\left\langle X, e_{j}\right\rangle=0 .
$$

In (4.7), letting $j=k$ and summing on $k$, we obtain

$$
\langle B x, X\rangle=0 \text {. }
$$

On the other hand, we find the $x * x$ component of (4.2), that is

$$
\langle B X, x\rangle=0 .
$$

Combining (4.9) with (4.8), we know that

$$
X\langle B x, x\rangle=0 .
$$

This means that $\langle B x, x\rangle$ is a constant. But we know that $\lambda(x)=2 n \alpha^{2}-\langle B x, x\rangle-$ $4(n+1)$ is also a constant. Thus $\alpha$ is a constant.

Then finding the $x * e_{n+1}$ component of (4.2), we have

$$
\left\langle B X, e_{n+1}\right\rangle=0 .
$$


But from (4.5), we also know that $\left\langle B x, e_{n+1}\right\rangle$ is a constant. Differentiating this relation and using (4.8) and (4.10), we obtain

$$
\left\langle B x, D_{X} e_{n+1}\right\rangle=0 .
$$

Now we find the $e_{r} * Y$ component of (4.2), that is

$$
\left\langle B x, e_{r}\right\rangle\langle X, Y\rangle+\left\langle h(X, Y), e_{r}\right\rangle=0 .
$$

Combining (4.11) with (4.12), we obtain

$$
\left\langle h(X, Y), D_{Z} e_{n+1}\right\rangle=0 .
$$

If there exists a tangent vector field $Z_{0}$, such that $D_{Z_{0}} e_{n+1} \neq 0$, we can choose $e_{n+2}$ parallel to $D_{z_{0}} e_{n+1}$. Then $\left\langle h(X, Y), e_{n+2}\right\rangle=0$ and $A_{n+2}=0$. This means that $M^{n}$ is contained in a totally geodesic submanifold of $S^{m-1}(1)$. This is a contradiction.

Thus for any $x \in M^{n}$ and $Z \in T_{x}\left(M^{n}\right), D_{z} e_{n+1}=0$. Then finding the $e_{r} * x$ component of $(4.2)$, we know that

$$
\left\langle B X, e_{r}\right\rangle=0,
$$

where $r=n+1, \ldots, m-1$.

Combining (4.14), (4.9) with $\langle B X, Y\rangle=\lambda\langle X, Y\rangle$, we know that $B X=\lambda X$, for any $X \in T_{x}\left(M^{n}\right)$ and any $x \in M^{n}$. By Lemma 4.4 , we can find $X_{1}, \ldots, X_{m} \in$ $\bigcup_{x \in M^{n}} T_{x}\left(M^{n}\right)$, which are linearly independent. Thus $B X_{i}=\lambda X_{i}, i=1, \ldots m$, and then $B V=\lambda V$, for any $V \in E^{m}$. Applying this relation in (4.5), we obtain $\alpha=0$. This means that $M^{n}$ is minimal.

Conclusion. Let $x: M^{n} \rightarrow S^{m-1}(1) \subset E^{m}$ be a submanifold of $S^{m-1}(1)$ whose quadric representation satisfies $\Delta \widetilde{x}=B \tilde{x}+C$. If it is full in $S^{m-1}(1)$, from Theorem 3.1 and Lemma 4.5 , we know that $n=m-1$ and $M^{n}$ is a piece of $S^{m-1}(1)$. If it is not full in $S^{m-1}(1)$, there must exist a positive integer $k<m$, such that $M^{n}$ is full in $S^{k-1}(1)$. Then we know that $n=k-1$ and $M^{n}$ is a piece of $S^{n}(1)$. That is to say $M^{n}$ is a totally geodesic submanifold of $S^{m-1}(1)$.

\section{REFERENCES}

[1] M. Barros and B.Y. Chen, 'Spherical submanifolds which are of 2-type via the second standard immersion of the sphere', Nagoya Math. J. 108 (1987), 77-91.

[2] B.Y. Chen, Total mean curvature and submanifolds of finite type (World Scientific, 1984).

[3] I. Dimitric, 'Quadric representation of a submanifold', Proc. Amer. Math. Soc. 114 (1992), 201-210. 
[4] J. Lu, 'Quadric representation of a submanifold with parallel mean curvature vector', Adv. in Math. (China) 25 (1996), 433-437.

[5] J. Lu, 'Hypersurface with special quadric representation', Bull. Austral. Math. Soc. 56 (1997), 227-234.

[6] J. Lu, 'Submanifolds whose Quadric representations satisfy $\Delta \widetilde{x}=B \widetilde{x}+C$ ', Kodai Math. J. 20 (1997), 135-142.

Division of Mathematics

National Institute of Education

Nanyang Technological University

Singapore 259756

e-mail: NF2363833U@ACAD21.NTU.EDU.SG 